\title{
Guillain-Barré Syndrome Triggered by COVID-19: A Pediatric Case Report and Literature Review
}

\author{
${ }^{1}$ Department of Neurology, University of Colorado School of Medicine, USA \\ ${ }^{2}$ Department of Pediatrics, University of Colorado School of Medicine, USA \\ ${ }^{3}$ Section of Pediatric Emergency Medicine, Children's Hospital Colorado, USA \\ ${ }^{4}$ Section of Pediatric Neurology, Denver Health, USA \\ ${ }^{5}$ Department of Neurology, Swedish Medical Center, USA \\ ${ }^{6}$ Section of Internal Medicine, Denver Health, USA \\ ${ }^{7}$ Department of Medicine, University of Colorado School of Medicine, USA \\ ${ }^{8}$ Section of Pediatric Radiology, Children's Hospital Colorado, USA \\ ${ }^{9}$ Department of Radiology, University of Colorado School of Medicine, USA \\ ${ }^{10}$ University of Colorado School of Medicine, USA \\ ${ }^{11}$ Section of Child Neurology, Children's Hospital Colorado, USA
}

Anthony Declusin, $M D^{1 *}$, Laura Bricklin, $M D^{2}$, Melisa Tanverdi, $M D^{2,3}$, Andrew White, $M D^{2,4}$ Michelle Brajcich, $M D^{2}$, John Szigety, $M D^{2}$, Lauren DiSalvo, $D O^{5}$, Nicholas Breitnauer, $M D^{6,7}$, Nicholas Stence, $M D^{8,9}$, William Mundo, $\mathrm{MPH}^{10}$, and Padmini Palat, MD, $\mathrm{MPH}^{2,11}$

\begin{abstract}
Coronavirus disease 2019 (COVID-19) has been associated with a variety of neurologic manifestations, including GuillainBarré syndrome (GBS), but few pediatric cases have been reported. We would like to present a pediatric case of GBS in association with a recent COVID-19 infection that presented with an isolated clinical facial nerve palsy.
\end{abstract}

\section{Keywords}

GBS, Miller-Fisher, Bell's palsy, Neuritis, Post-COVID

\section{Abbreviations}

SARS-CoV-2: Severe Acute Respiratory Syndrome Coronavirus 2; COVID-19: Coronavirus Disease 2019; GBS: Guillain-Barré Syndrome; CT: Computed Tomography; MRI: Magnetic Resonance Imaging; CSF: Cerebrospinal Fluid; EMG/NCS: Electromyography and Nerve Conduction Studies; IVIG: Intravenous Immunoglobulin

\section{Introduction}

The severe acute respiratory syndrome-coronavirus type 2 (SARS-CoV-2) began to appear in December of 2020. Since onset, it has infected more than 100 million people and is responsible for nearly 2.5 million deaths worldwide [1]. While the coronavirus disease 2019 (COVID-19) is primarily a respiratory illness, the viral infection has been associated with complications in multiple other organ systems, including both the central and peripheral nervous systems. COVID-19 infection has been associated with various neurologic manifestations, including stroke, meningoencephalitis, hyposmia, hypogeusia, Guillain-Barré syndrome (GBS), and cranial nerve palsies among several others [2]. Many of these complications have been documented in adult patients, but few have been reported in pediatric populations due to a limited number of documented symptomatic infections.
*Corresponding author: Anthony Declusin, MD, Department of Neurology, University of Colorado School of Medicine, Aurora, CO, 80045 USA
Accepted: August 07, 2021
Published online: August 09, 2021
Citation: Declusin A, Bricklin L, Tanverdi M, et al. (2021) Guillain- Barré Syndrome Triggered by COVID-19: A Pediatric Case Report and Literature Review. J Pediatr Neurol Neurosci 5(2):145-149 


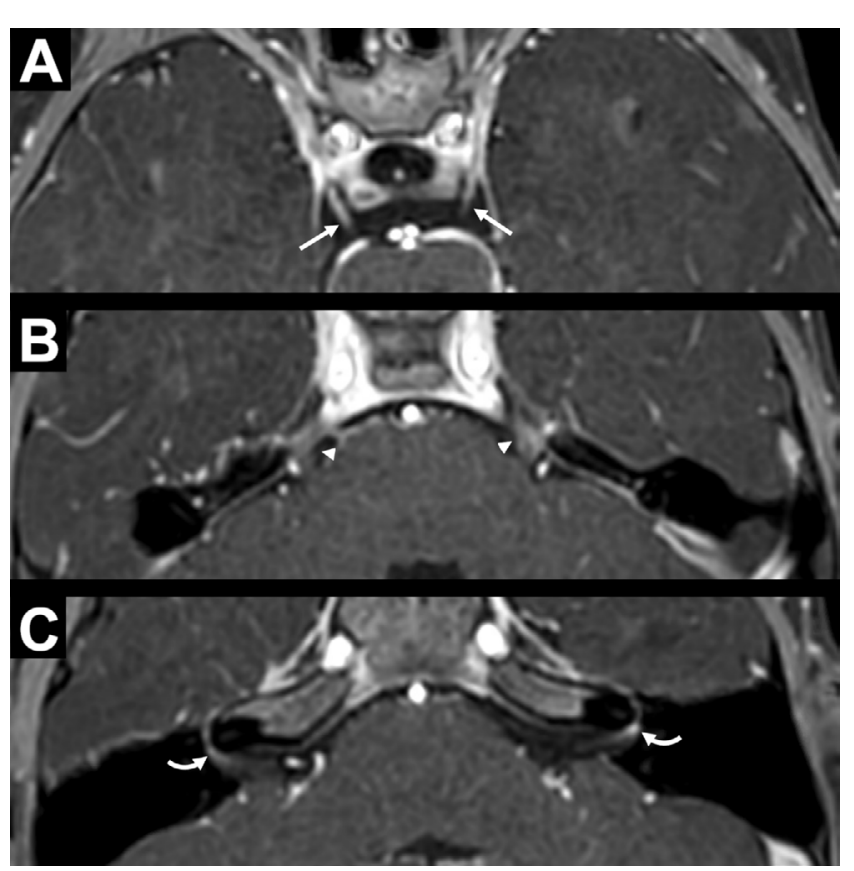

Figure 1: MRI Brain Demonstrating Multiple Cranial Nerve Enhancement.

Axial high resolution post-contrast $\mathrm{T} 1$ weighted images demonstrate levels of abnormal cranial nerve enhancement, including bilateral $\mathrm{CN}$ III (A, arrows), left more than right $\mathrm{CN} \vee(\mathrm{B}$, arrowheads), and left more than right CN VII (C, curved arrows).

We present a case of a 2-year-old girl with a positive COVID-19 polymerase chain reaction (PCR), who presented with a left facial nerve palsy and associated imaging abnormalities ultimately and was subsequently found to have GBS based upon clinical and laboratory criteria.

\section{Case Report}

A 2-year-old female with a history of constipation presented to our pediatric emergency department with acute onset of left facial droop and abnormal gait beginning approximately two hours prior to arrival. The mother of the child reported that the child had also experienced left arm pain, a change in voice, less oral intake, and ringing in her ears the day prior to presentation. Past medical history was notable for a COVID-19 infection with mild congestion, cough, and fatigue approximately six weeks prior to presentation, but there were no acute infectious symptoms or fever in the days leading up to presentation.

On initial examination, she was noted to be awake and fussy with tachycardia and elevated blood pressure. Her neurologic exam was notable for a left-sided facial droop with inability to raise her left eyebrow, flattening of the nasolabial folds, and asymmetric smile. The remainder of her cranial nerve exam was within normal limits. She had full active range of motion, strength, sensation, and reflexes in all four extremities. On examination of gait, it was notable that she refused to bear weight on the ball of her left foot and instead put all the weight on her left heel. The remainder of her physical exam was within normal limits.
Given the acute onset of her neurologic deficits of the left face and possible lower extremities, a stroke alert was called, which expedited her time to imaging. Computed tomography (CT) of the head without contrast showed no abnormalities. Sedated magnetic resonance imaging (MRI) of the brain with and without contrast demonstrated concern for diffuse cranial neuritis with enhancement of multiple cranial nerves, but no evidence of stroke (Figure 1). Details of laboratory and imaging findings are described below.

Complete blood count was notable for a leukocytosis to $11.5^{*} 103 /$ uL with an elevated absolute lymphocyte count (5250/uL) and elevated absolute eosinophil count of $0.3^{*}$ 103/uL. Comprehensive metabolic panel showed a metabolic acidosis with a bicarbonate of $15 \mathrm{mmol} / \mathrm{L}$ and an anion gap of 14, normal electrolytes and glucose, as well as blood urea nitrogen, creatinine, and liver function tests. Coagulation panel (prothrombin time, partial thromboplastin time, international normalized ratio, D-dimer, and fibrinogen) was within normal limits. Inflammatory markers were normal with a C-reactive protein of $<0.5 \mathrm{mg} / \mathrm{dL}$ and erythrocyte sedimentation rate of $10 \mathrm{~mm} / \mathrm{hr}$. Respiratory pathogen panel was positive for SARS-CoV-2 RNA and no other organisms. Electrocardiogram showed normal sinus rhythm. CT of the head without contrast demonstrated no acute intracranial abnormality. MRI of the brain with and without contrast and with angiogram showed asymmetric, avid left facial nerve enhancement at the fundus of the internal acoustic canal (IAC), as well as more faint enhancement of other cranial nerves including bilateral cranial nerves three, five, and possibly right facial nerve at the IAC fundus. There was also equivocal mild diffuse sulcal enhancement felt to be non-specific and likely related to sedation that was required to obtain study; correlation with cerebrospinal fluid (CSF) testing was recommended. Lumbar puncture was performed, and CSF analysis revealed 1 white blood cell / mm*3, 450 red blood cells / mm*3, glucose of $59 \mathrm{mg} / \mathrm{dL}$, and protein of 46 $\mathrm{mg} / \mathrm{dL}$. On CSF meningitis encephalitis panel, no organisms were detected, and there was no growth on bacterial culture.

The patient was admitted to hospital medicine for observation. She had persistent left lower motor neuron facial nerve palsy on exam the following morning without other notable neurologic deficits. The patient was discharged on oral prednisolone $1 \mathrm{mg} / \mathrm{kg}$ for seven days with plans to follow-up in neurology clinic.

Three weeks after hospital discharge, the patient returned to the emergency department for refusal to bear weight since the prior admission. She was also noted to have new constipation and complained of unlocalizable back and bottom pain. Initial laboratory work-up revealed leukocytosis (12.4 *103/uL) with elevated absolute lymphocyte count (6000/uL), normal c-reactive protein, erythrocyte sedimentation rate, creatine kinase, and electrolytes. Physical examination was significant for refusal to sit upright or bear weight on bilateral lower extremities, generalized hypotonia, 4-/5 strength of all extremities, intact sensation to mild noxious stimuli, absent patellar and Achilles deep tendon reflexes, and no reproducible pain to palpation of back or lower extremities with full passive range of motion. 
Citation: Declusin A, Bricklin L, Tanverdi M, et al. (2021) Guillain-Barré Syndrome Triggered by COVID-19: A Pediatric Case Report and Literature Review. J Pediatr Neurol Neurosci 5(2):145-149

Radiographs of bilateral hips, tibia, and fibula were negative for fracture, dislocation, or bony abnormalities to suggest avascular necrosis. Magnetic resonance imaging of the cervical, thoracic, and lumbar spine with and without contrast was normal. Lumbar puncture was performed, and CSF revealed no red blood cells or white blood cells, normal glucose, and elevated protein $(152 \mathrm{mg} / \mathrm{dL})$. Based on the constellation of hypotonia, areflexia, and albuminocytologic dissociation, the patient was diagnosed with GBS. A serum ganglioside antibody panel was also sent at the time of lumbar puncture and later resulted with elevated GM2 IgG/ IgM antibodies (54 IV, upper limit of normal 50 IV).

The patient was treated with intravenous immunoglobulin (IVIG) (2 g/kg divided over two days) and a pain regimen of gabapentin, acetaminophen, and ibuprofen. She received physical and occupational therapy as well as a bowel regimen of sennosides and polyethylene glycol. By the fourth day of hospitalization, she was able to sit unsupported, briefly stand independently, and smile and engage in play. Her hypotonia improved some but her deep tendon reflexes remained absent. The patient experienced no clinical evidence of cardio respiratory dysfunction throughout hospitalization and was discharged home with ongoing outpatient therapies and neurology follow-up.

The patient was seen for follow-up electromyography and nerve conduction studies (EMG/NCS) about one month following discharge. At that time, the patient was able to ambulate on her own for short distances and was still working with physical therapy. EMG/NCS findings were suggestive of a severe sub acute sensorimotor poly neuropathy with predominantly demyelinating features with secondary axonal loss, consistent with a diagnosis of GBS.

\section{Discussion}

Para-/post-infectious neuropathies are an unfortunate complication of a variety of different bacterial and viral infections. Para-infectious neuropathies may be caused by direct nerve injury from pathogen invasion or by an antibodymediated response, while post-infectious neuropathies are believed to be exclusively caused by an antibody-mediated response [3]. These antibody-mediated responses are believed to develop by molecular mimicry via cross-reactivity of an autologous antigen with a pathogenic antigen [4,5]. The most common clinical manifestations of presumed para-/ post-infectious neuropathies are Bell's palsy and GBS.

Bell's palsy is an idiopathic self-resolving lower motor neuron facial nerve palsy $[5,6]$. This is classically believed to be triggered by viral reactivation of latent herpes simplex virus type-1 (HSV-1) or varicella-zoster virus (VZV), causing direct nerve injury. However, additional viral infections have been associated with an immune-mediated response, similar to GBS [6]. Steroids are the recommended treatment and increase the probability of recovery. Addition of antivirals may help increase the likelihood of recovery, but the evidence is more mixed [5].

GBS is typically a post-infectious acute immune-mediated neuropathy that presents days to weeks following the antecedent infection, most commonly due to Campylobacter jejuni $[3,4]$. GBS has a wide variety of variants that differ in neurologic distribution, progression, neurophysiologic findings, and antibodies. Antibodies are frequently directed at gangliosides present on myelin sheaths at the nodes of Ranvier along nerve axons that result in demyelination, and the specific auto antibodies can help define the clinical variant of GBS. For example, Miller-Fisher syndrome (MFS) is classically characterized by the presence of anti-GQ1b antibodies in $\sim 85 \%$ of cases. Neurophysiologic findings on EMG/NCS can define the neuropathy as either demyelinating or axonal, which can help clarify the variant, as well. CSF is classically characterized by an albuminocytologic dissociation, which is an elevated protein in the absence of pleocytosis [4]. However, the protein elevation may take up to 3 weeks to appear, and a pleocytosis can be present $1<50$ white blood cells) [7]. Patients are typically treated with IVIG or plasmapheresis. Multiple clinical trials have no shown benefit of steroids [4].

Various cranial neuropathies have been associated with COVID-19 infections in several documented cases, most frequently involving the facial nerves [8-22]. These cranial neuropathies can be either symptomatic based upon abnormal clinical exam or asymptomatic with only abnormal enhancement seen on MRI $[2,9,13]$. Many of these cranial neuropathies have been reported in adult patients, but only a few have been documented in pediatric patients [8-11,14,19].

Cranial neuropathies have occurred in the setting of either acute or recent COVID-19 infections, which have ranged from asymptomatic to severe infections $[9,11,14,19,22]$. Isolated cranial neuropathies have been documented to occur 3-7 days following onset of COVID-19 infectious symptoms [10]. Cranial neuropathies have occurred in isolation and/or associated with additional neurologic symptoms, including GBS $[8,10,11,13,14]$. Steroids, antivirals, and/or IVIG have been trialed as treatments for isolated cranial neuropathies with reported improvement in most patients, but at least one patient was documented to recover without treatment $[8,10,11,13,14,19]$.

Similarly, various forms of GBS have also been associated with COVID-19 infections [2,9,10,12,13,15-18,21,23]. These have included the classic acute inflammatory demyelinating poly neuropathy (AIDP), MFS, acute motor sensory axonal neuropathy (AMSAN), acute motor axonal neuropathy (AMAN), and other less common mixed variants $[2,10,12,13,15,16,18,20,23]$. Some of these cases have been associated with cranial neuropathies, mainly facial nerve palsies, that typically develop following the onset of extremity symptoms, but can more rarely be the only initial presenting neurologic symptom of GBS $[2,10,12,13,15-18,21,23]$. Many cases have been documented in adult patients, but only a few are reported in children $[18,20,23]$.

These GBS cases have occurred in acute or recent COVID-19 infections, which have ranged from asymptomatic to severe infections $[12,15,18,20,21,23]$. GBS manifestations have been witnessed in patients 2-33 days following the onset of COVID-19 infectious symptoms, but in rare cases, GBS 
Citation: Declusin A, Bricklin L, Tanverdi M, et al. (2021) Guillain-Barré Syndrome Triggered by COVID-19: A Pediatric Case Report and Literature Review. J Pediatr Neurol Neurosci 5(2):145-149

symptoms can precede the onset of the primary symptoms of COVID-19 by up to 8 days $[2,10,12,13,15,17,18,21]$.

Lumbar puncture results have shown the classic albuminocytologic dissociation in most, but protein has been documented as normal in a smaller subset of patients $[2,10,12,13,16-18,20,21,23]$. For those with a normal protein, it is possible that the LPs may have been performed early in the onset of GBS prior to development of an elevation in CSF protein. Mild pleocytosis and/or oligoclonal bands have been documented in the CSF in a small number of patients $[15,18]$. Ganglioside antibody results have been absent in most patients when tested, but have shown positive antiGD1b or anti-GM1 in a few cases $[2,12,15-18,20]$. MRI results are commonly reported as normal in most patients, but have shown spinal nerve root enhancement, enhancement of affected cranial nerves, and/or more rarely, brainstem/spinal cord leptomeningeal enhancement $[2,9,16,18,20]$.

GBS has progressed to requiring mechanical ventilation in a small percentage of patients, but a portion of these cases may have been complicated by primary lung disease secondary to SARS-CoV-2 $[2,10,13,16-18,23]$. GBS has been treated with IVIG, plasmapheresis, and/or steroids with expected responses based upon prior treatment data, but in one documented case, the patient recovered without any treatment $[10,12,13,15,17,18,21,23]$.

The pathogenesis of these specific neurologic complications appears to be consistent with an immunemediated process based upon the available data to date. Additional neurologic complications of COVID-19 infection include anosmia and dysgeusia, which have been postulated by some to be caused by direct viral invasion of associated central nervous system (CNS) anatomic sites. However, evidence of direct viral invasion of the CNS has been limited to date $[2,13]$.

Our patient initially presented a clinical facial nerve palsy and abnormal gait involving the left lower extremity. She had tested positive for COVID-19 about three weeks prior, but her infection remained asymptomatic. Her COVID-19 PCR remained positive through her initial presentation. She had imaging findings supportive of her cranial neuropathy with active enhancement of the left facial nerve. However, she also had asymptomatic enhancement of multiple additional cranial nerves. She was discharged with oral prednisolone treatment, consistent with standard-of-care treatment for Bell's palsy. The oral prednisolone did resolve her facial nerve palsy, but she continued to have progressive extremity weakness that was not appreciated on her initial presentation. Ultimately, this led to her representation and diagnosis of GBS based upon her clinical exam and CSF profile, consistent with an albuminocytologic dissociation. The patient also was found to have positive anti-GM2 ganglioside IgG antibodies present in the serum, which has been seen in GBS caused by cytomegalovirus (CMV) infection in addition to multifocal motor neuropathy (MMN) [4]. She began to recover with IVIG treatment.

Despite the patient initially presenting with a presumed Bell's palsy, the patient very likely had GBS all along. The timing and presentation are consistent with a GBS variant, which has been seen in multiple other cases associated with COVID-19 infection [2,9,10,12,13,15-18,20,21,23]. Similarly, isolated cranial nerve palsies have been seen as the only presenting symptom of COVID-19-associated GBS in a small number of cases $[2,10,12,13,15-18,20,21,23]$. The patient's symptomatic and asymptomatic cranial nerve enhancement on MRI has also been associated with other COVID-19 cases $[2,9,13]$. It is unclear how many of these cases occurred with solely isolated cranial neuropathies and/or were diagnosed with GBS.

Her facial nerve palsy was not the only notable abnormality on her exam on her initial presentation. The patient was additionally presenting with extremity symptoms, described as an antalgic gait of her left lower extremity. At that time, she did not have any clear signs of weakness on her initial exam. This was likely because her GBS had not significantly impaired her extremity strength enough to be detectable on routine neurologic examination. The weakness became more evident as her disease course progressed and was noted on confrontational testing at the time of her representation. Her initial CSF profile upon presentation did not have an albuminocytologic dissociation, but this was likely due to her early presentation in her GBS disease course. She very clearly had an albuminocytologic dissociation upon her representation a few weeks later. Additionally, although her facial nerve palsy improved with oral prednisolone, the fact that the remainder of her symptoms continued to progress despite steroid treatment, further supports her diagnosis of GBS.

This case exemplifies the diagnostic and treatment challenges of COVID-19 associated neurologic symptoms and GBS variants, in general. Vigilance is required upon initial evaluation of new neurologic symptoms and a thorough neurologic examination should be performed to ensure an accurate diagnosis is achieved.

No matter their location or distribution, acute to sub acute onset of neuropathies in the setting of acute or recent COVID-19 infection should be considered as a possible firstsign of GBS in these patients. Additionally, multiple cranial nerve enhancement may be indicative of the same, or at the very least, warrant further workup and monitoring. Additional case series may help to further define the full spectrum of COVID-19 associated GBS.

\section{Conclusion}

GBS appears to be associated with COVID-19 infection in all age groups, but so far has been reported mostly in adults. It is unclear if recent COVID-19 infection triggered GBS in our patient, but there appears to be an association based upon prior case reports. Several GBS variants have been associated with COVID-19 infection, and this diagnosis should be considered in patients presenting with acute to sub acute onset of neuropathies in the setting of acute or recent COVID-19 infection. Additionally, multiple cranial nerve enhancement on MRI may indicate GBS in patients with COVID-19. 


\section{References}

1. WHO (2021) WHO Coronavirus Disease (COVID-19) Dashboard.

2. Koralnik IJ, Tyler KL (2020) COVID-19: A Global Threat to the Nervous System. Ann Neurol 88: 1-11.

3. England J (2019) Para infectious neuropathies. J Neurol Sci 405 22.

4. Bourque PR, Chardon JW, Massie R (2015) Autoimmune peripheral neuropathies. Clin Chim Acta 449: 37-42.

5. Reich SG (2017) Bell's Palsy. Contin Minneap Minn 23: 447-466.

6. Zhang W, Xu L, Luo T, et al. (2020) The etiology of Bell's palsy: A review. J Neurol 267: 1896-1905.

7. Fokke C, van den Berg B, Drenthen J, et al. Diagnosis of GuillainBarré syndrome and validation of Brighton criteria. Brain J Neurol 137: 33-43.

8. Codeluppi L, Venturelli F, Rossi J, et al. (2021) Facial palsy during the COVID-19 pandemic. Brain Behav 11: e01939.

9. Lindan CE, Mankad K, Ram D, et al. (2020) Neuroimaging manifestations in children with SARS-CoV-2 infection: a multinational, multicentre collaborative study. Lancet Child Adolesc Health 5: 167-177.

10. Valiuddin HM, Kalajdzic A, Rosati J, et al. (2020) Update on Neurological Manifestations of SARS-CoV-2. West J Emerg Med $21: 45-51$.

11. Wan Y, Cao S, Fang Q, et al. (2020) Coronavirus disease 2019 complicated with Bell's palsy: A case report. Research Square.

12. Gutiérrez-Ortiz C, Méndez-Guerrero A, Rodrigo-Rey $S$, et al. (2020) Miller Fisher syndrome and polyneuritis cranialis in COVID-19. Neurology 95: e601-e605.

13. Paliwal VK, Garg RK, Gupta A, et al. (2020) Neuromuscular presentations in patients with COVID-19. Neurol Sci 41: 3039-3056.
14. Wei $H$, Yin $H$, Huang $M$, et al. (2020) The 2019 novel cornoavirus pneumonia with onset of oculomotor nerve palsy: A case study. J Neurol 267: 1550-1553.

15. https://pubmed.ncbi.nlm.nih.gov/32468450/

16. Toscano G, Palmerini F, Ravaglia S, et al. (2020) Guillain-Barré syndrome associated with SARS-CoV-2. N Engl J Med 382: 25742576.

17. Manganotti P, Bellavita G, D'Acunto L, et al. (2021) Clinical neurophysiology and cerebrospinal liquor analysis to detect Guillain-Barré syndrome and polyneuritis cranialis in COVID-19 patients: A case series. J Med Virol 93: 766-774.

18. Abu-Rumeileh S, Abdelhak A, Foschi M, et al. (2020) GuillainBarré syndrome spectrum associated with COVID-19: an up-todate systematic review of 73 cases. J Neurol 268: 1133-1170.

19. Theophanous C, Santoro JD, Itani R (2021) Bell's palsy in a pediatric patient with hyper IgM syndrome and severe acute respiratory syndrome coronavirus 2 (SARS-CoV-2). Brain Dev 43: 357-359.

20. Khaja M, Gomez GPR, Santana Y, et al. (2020) A 44-year-old hispanic man with loss of taste and bilateral facial weakness diagnosed with Guillain-Barré syndrome and bell's palsy associated with sars-cov-2 infection treated with intravenous immunoglobulin. Am J Case Rep 21:e927956.

21. Agosti E, Giorgianni A, D'Amore F, et al. (2021) Is Guillain-Barrè syndrome triggered by SARS-CoV-2? Case report and literature review. Neurol Sci 42: 607-612.

22. Pan S, Chen WC, Baal JD, et al. (2020) Neuroradiological Features of Mild and Severe SARS-CoV-2 Infection. Acad Radiol 27: 1507-1514.

23. Curtis M, Bhumbra S, Felker MV, et al. (2020) Guillain-Barré syndrome in a child with COVID-19 Infection. Pediatrics 147: e2020015115.

DOI: $10.36959 / 595 / 427$

Copyright: (C) 2021 Declusin A. This is an open-access article distributed under the terms of the Creative Commons Attribution License, which permits unrestricted use, distribution, and reproduction in any medium, provided the original author and source are credited. 